\title{
Characterization of highly charged titanium ions produced by nanosecond pulsed laser
}

\author{
S.A. ABBASI,${ }^{1,2}$ M.S. HUSSAIN,${ }^{1}$ B. ILYAS, ${ }^{3}$ M. RAFIQUE, ${ }^{2}$ A.H. DOGAR, ${ }^{1}$ AND A. QAYYUM ${ }^{1}$ \\ ${ }^{1}$ Electron LINAC Project, Pakistan Institute of Nuclear Science and Technology, Nilore, Islamabad, Pakistan \\ ${ }^{2}$ Department of physics, University of Azad Jammu \& Kashmir, Muzaffarabad, Pakistan \\ ${ }^{3}$ Optics Laboratories, Islamabad, Pakistan \\ (Received 30 August 2014; ACCEPTED 8 November 2014)
}

\begin{abstract}
In this work, plasma is produced by irradiating a Ti target with $10 \mathrm{~ns}$ pulsed Nd:YAG $(\lambda=1064 \mathrm{~nm})$ laser. The laser fluence at the target was varied in the range of $2-20.3 \mathrm{~J} / \mathrm{cm}^{2}$. The ion signal from freely expanding Ti plasma in vacuum was characterized with the help of ion collector and time-of-flight electrostatic energy analyzer. The ion charge state was found to increase with the laser fluence and maximum available ion charge in this fluence range is $\mathrm{Ti}^{4+}$. A correlation between the intensities of various ion charge states was observed, which indicates that higher charge states are most probably produced through stepwise ionization mechanism. It is also observed that charge state distribution of plasma can be controlled by variation of the laser fluence. In addition, energy distribution of ion charge states $\mathrm{Ti}^{n+}(n=1-4)$ is measured by varying back plate voltage of the electrostatic energy analyzer for a fixed laser fluence of $20.3 \mathrm{~J} / \mathrm{cm}^{2}$. Ions energy distributions were in the range of $0.36-3.0 \mathrm{keV}$ and the most probable ion energy was found to increase linearly with ion charge state. The estimated equivalent potential at the laser fluence of $20.3 \mathrm{~J} /$ $\mathrm{cm}^{2}$ is about $310 \mathrm{~V}$. These results are in good agreement with the predictions of electrostatic model of ion acceleration in laser plasma.
\end{abstract}

Keywords: Energy distribution; Laser fluence; Laser-produced plasma; Titanium; Time-of-flight electrostatic energy analyzer

\section{INTRODUCTION}

Intense nanosecond laser radiation focused on to a solid target induces thermal processes that lead to localized melting and vaporization of material from the irradiated area of the solid. At the same time laser radiation interacts with expanding plasma and high charge states are produced by electron-ion collisions and multi-photon interaction processes. After production the plasma expands at supersonic velocity, and self-generated electric field in the plume determines the energy and flow of the ions in vacuum. This non-isotropic electric field is directed mainly along the target surface normal and its duration is of the order of laser pulse width. This localized plasma has found increasing amount of interest because of its wide range of applications in various applied and research fields, such as production of highly charged ions for accelerators and ion implanters (Láska et al., 1996; Burdt et al., 2010), inertial confinement in

Address correspondence and reprint requests to: Abdul Qayyum, Electron LINAC Project, Pakistan Institute of Nuclear Science and Technology, Nilore, Islamabad, Pakistan. E-mail: aqayyum11@yahoo.com fusion (Lindl, 1995), high intensity and high energy ion accelerators (Haseroth \& Hora, 1996; Krasa et al., 1998; Collier et al., 1996), material modification (Trtica et al., 2013) and synthesis of nano-particles (Riabinina et al., 2010).

It has been reported that the plasma temperature, ion and electron densities, ion energy, and charge state distribution depends strongly on the parameters of the incident laser photon such as its wavelength, pulse duration, spot size, and laser fluence (Nassisi et al., 2002; Lunney, 1995; Jordan et al., 1995; Torrisi et al., 2003). But it appears that only a few studies on the effect of laser fluenec on the ion charge state, energy distribution of various charge states (Láska et al., 1996; Ilyas et al., 2011), and estimation of electric field in laser produced plasma are available in literature (Caridi et al., 2010; Torrisi et al., 2008a). These data are necessary for a fundamental understanding of laser-generated plasmas and their application in the development of high current and high charge state ion sources for conventional ion accelerators. In addition, these data are also quite important for the community of scientists working on pulsed laser deposition of titanium and various other metal thin films because 
the flux, charge state and energy distribution of the ions arriving on the growing film has major influence on the properties of the film (Mostako \& Khare, 2012). Thin films of titanium and its alloys are widely used in several industries, such as aerospace engineering, motor science, chemical engineering sectors due to its very high specific strength, and remarkable corrosion resistance (Itoh et al., 1999; Komotori et al., 2001; Kobayash et al., 1998). Titanium has also been used as a functional thin film layer, like diffusion barriers, buffer layers, and interlayers, etc., in semiconductor devices (Cho et al., 2006; Lee et al., 2005).

Here in this study ion collector and time-of-flight (TOF) ion energy analyzer is used to investigate the fundamental properties of $\mathrm{Ti}$ ions produced by nanosecond pulsed laser ablation. In particular, we systematically investigated the effect of laser fleunce on the intensity and charge state of Ti ions. We also measured energy distribution of the various Ti ion charge states for a fixed value of the laser fluence and estimated equivalent accelerating potential in nonequilibrium laser plasma.

\section{EXPERIMENTAL SETUP}

The experimental setup consists of Nd:YAG laser with wavelength of $1064 \mathrm{~nm}$ and $10 \mathrm{~ns}$ pulse duration along with associated beam delivery optics, ablation chamber, TOF ion collector, electrostatic energy analyzer (EEA), detector and data acquisition system. The schematic diagram of experimental setup is shown in Figure 1. Pulsed laser beam is focused by an optical lens of focal length of $50 \mathrm{~cm}$ onto the surface of a $99.99 \%$ pure titanium target. The laser beam enters the vacuum chamber through a quartz window and falls on the target at an angle of $45^{\circ}$ with respect to target surface normal. A dc motor continuously rotates the target so that each laser shot strikes the fresh surface. A cylindrical IC of $10 \mathrm{~mm}$ aperture is placed at a distance of $6.5 \mathrm{~cm}$ from the target. IC is biased with $-50 \mathrm{~V}$ to repel plasma electrons. The signal from IC is obtained with the help of digital oscilloscope (LecRoy $500 \mathrm{MHz}$ ) from the

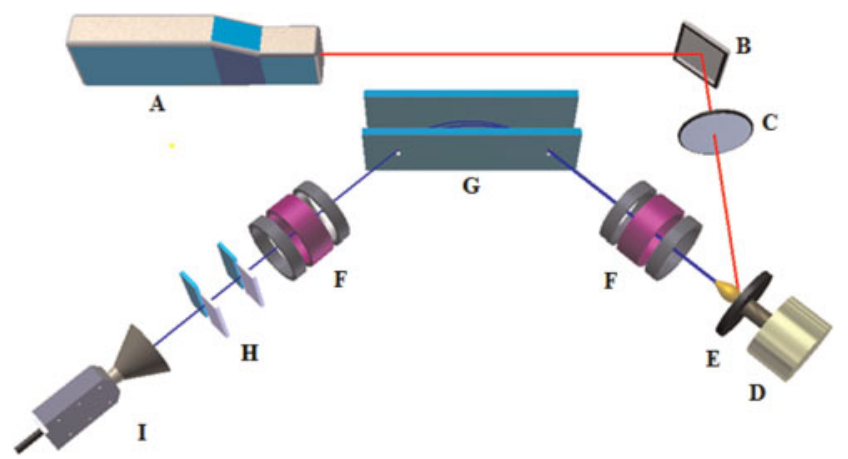

Fig. 1. Schematic of indigenously developed experimental facility (A) Nd:YAG laser, (B) Reflecting mirror, (C) Focusing lens, (D) dc motor, (E) Target, (F) Einzel lens, (G) $E / q$ filter (H) Slits, (I) channel electron multiplier. voltage drop across $1 \Omega$ resistor. A fast photodiode is used to trigger the oscilloscope at the time laser hits the target. The area of laser spot on the target was $0.65 \mathrm{~mm}^{2}$ and laser fluence was in the range of $2-20.3 \mathrm{~J} / \mathrm{cm}^{2}$.

The entrance slit of parallel plate ion energy analyzer is placed at a drift distance of $45 \mathrm{~cm}$ from the target. This distance is within the recommended range of drift distance for laser ion sources (Shirkov et al., 1995). The drift distance is necessary to reduce plasma effects such as ion recombination, plasma shielding, and arcing. Distance between two parallel plates of electrostatic energy analyzer is $35 \mathrm{~mm}$. The front plate is grounded and potential $V_{a}$ is applied on the back plate. Ions enter through the entrance slit of $2 \mathrm{~mm}$ width and then decelerated by electric field $E=-V_{a} / d$ between the two plates. In these configuration ions of specific energy to charge ratio, $E / q$, pass through the exit slit of $2 \mathrm{~mm}$ width. The detailed description of this device is given elsewhere (Ilyas et al., 2011). The ions are detected by channel electron multiplier (CEM) (Dr. Sjuts, type KBL5RS) and its signal is acquired by fast digital storage oscilloscope (LecRoy, $500 \mathrm{MHz}$ ). The total flight distance from the target to CEM is $1.31 \mathrm{~m}$. This whole arrangement provides energy resolved TOF spectra of the positively charged ions ablated by laser shot. The Einzel lenses placed before and after EEA are used to optimize the signal of the specific ion charge state, but these Einzel lenses are placed at ground potential during the study. The experiments are conducted in vacuum below $10^{-6}$ torr to minimize the charge exchange with ambient.

\section{RESULTS AND DISCUSSION}

A typical Ti ion pulse measured by IC for a laser shot of fluence $7.4 \mathrm{~J} / \mathrm{cm}^{2}$ is shown in Figure 2. For this measurement, IC was placed along the target surface normal at a distance of $6.5 \mathrm{~cm}$ from the target. It can be seen that ion current attains a

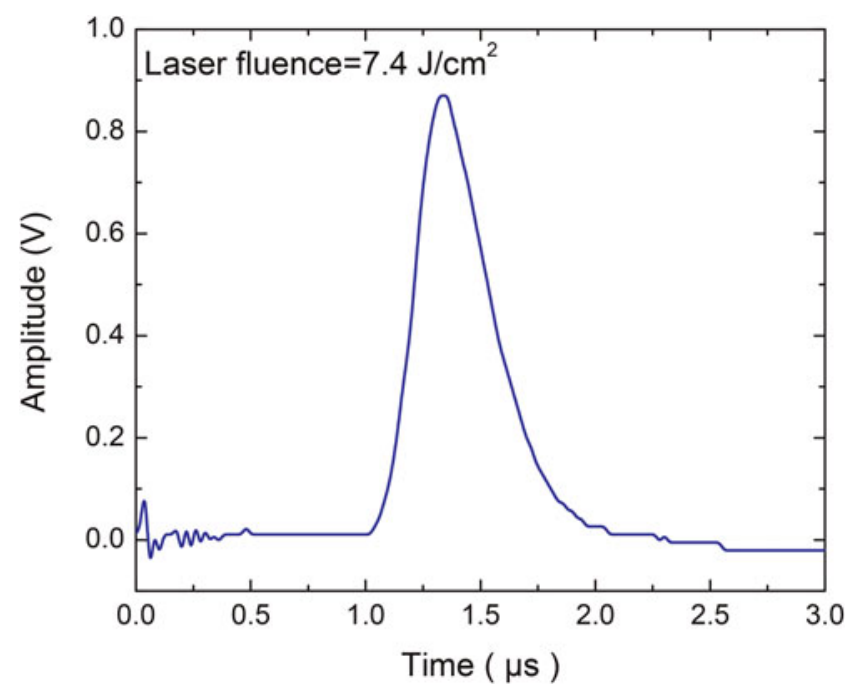

Fig. 2. TOF ion signal measured by IC placed at a distance of $6.5 \mathrm{~cm}$ along the target surface normal. 

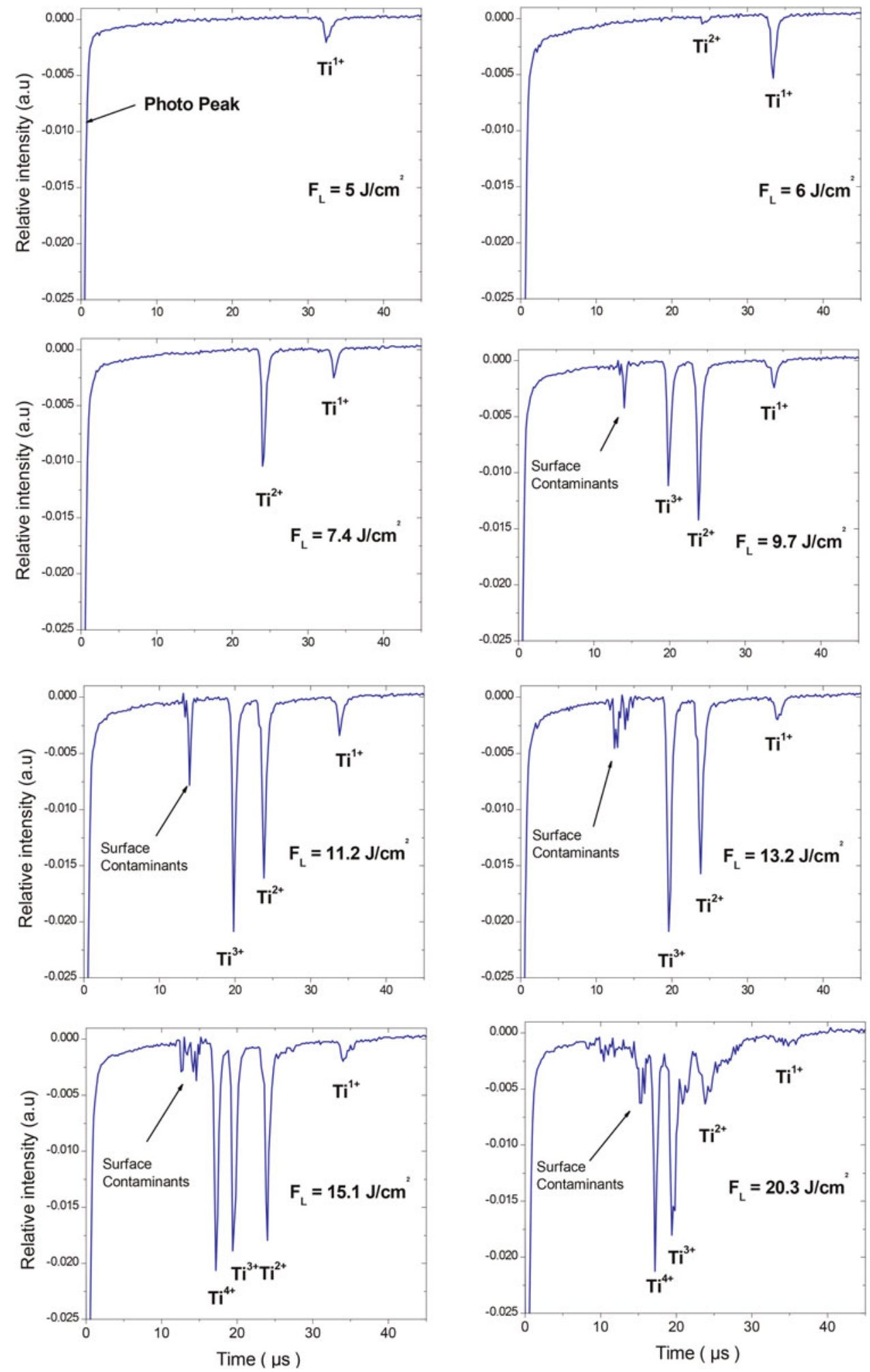

Fig. 3. TOF electrostatic energy analyzer (TOF-EEA) spectra of Ti ions for various values of the laser fluence. For these measurements filtering criterion of the ion energy analyzer was fixed at $E / q=502 \mathrm{eV} /$ charge.

maximum value and then decreases rather slowly as the plume continues to expand beyond the ion collector. In addition, ion current peak is fairly wide. These trends are in line with the recently reported results for $\mathrm{Cu}$ target in the fluence range (1.24 to $8.52 \mathrm{~kJ} / \mathrm{cm}^{2}$ ) (Yeates et al., 2010) and for $\mathrm{Ag}$ target in the fluence range $\left(1.5\right.$ to $7.5 \mathrm{~J} / \mathrm{cm}^{2}$ ) (Mannion et al., 2005). Relatively steep leading edge of ion pulse is composed of fast ions, which are most probably highly charged. 
The trailing edge of the ion pulse is composed of singly charged ions, which are less energetic as compared to highly charge ions because electric field in non-equilibrium plasma (Demtroder et al., 1970) accelerates ions proportional to their charge state. Therefore, broadness of the ion pulse (see Fig. 2) is attributed to the presence of various charge states of Ti ions in the laser produced plasma.

Then energy resolved TOF measurement were performed to determine $\mathrm{Ti}$ ions charge state distribution. In particular, we have systematically investigated the effect of laser fluence on the intensity and charge state distribution of $\mathrm{Ti}$ ions. Figure 3 shows measured energy resolved TOF spectra for the range of laser fluence (5.0 to $20.3 \mathrm{~J} / \mathrm{cm}^{2}$ ). The filtering criteria of electrostatic energy analyzer were fixed at $E$ / $q=502 \mathrm{eV} /$ charge for this measurement. The initial intense peak in every spectrum is photo peak which is due to the ultraviolet radiation reflected from the back plate of EEA on to the detector. Ion signal below the laser fluence of $5 \mathrm{~J} / \mathrm{cm}^{2}$ was not observed. At the laser fluence of $5 \mathrm{~J} / \mathrm{cm}^{2}$ only $\mathrm{Ti}^{1+}$ is observed (see Fig. 3). Ti ions could not be detected below the laser fluence of $5 \mathrm{~J} / \mathrm{cm}^{2}$. It is important to note that reported laser ablation threshold of $\mathrm{Ti}$ is $4.5 \mathrm{~J} / \mathrm{cm}^{2}$ (Vlădoiu et al., 2008). The ion $\mathrm{Ti}^{2+}$ appeared at $6 \mathrm{~J} / \mathrm{cm}^{2}$ and became more intense as compared to $\mathrm{Ti}^{1+}$ at $7.4 \mathrm{~J} /$ $\mathrm{cm}^{2}$. As the laser energy is further increased $\mathrm{Ti}^{3+}$ and then $\mathrm{Ti}^{4+}$ also appeared. It is noted that at the laser fluence of $9.3 \mathrm{~J} / \mathrm{cm}^{2}$, some additional ions appeared whom TOF does not match with the calculated flight time of Ti ions. These ions are most probably due to surface contaminants. The relative intensity of various ion charge states measured as a function of laser fluence is shown in Figure 4. Following trends can be deduced from the Figures 3 and 4. (1) Each charge state seems to have appearance threshold fluence, which indicates that exact appearance threshold fluence for every charge state can be found by fine tuning of the laser fluence. (2) The maximum available ion charge state increases with the laser fluence, indicating that ion charge distribution of the laser plasma can be controlled by laser fluence. (3) The intensity of the charge states $\mathrm{Ti}^{n+}(n=1,2,3)$ rises to a maximum value and then decreases with the further increase of laser fluence. (4) As the charge state $\mathrm{Ti}^{n+}$ appears the intensity of charge state $\mathrm{Ti}^{(n-1)+}$ starts decreasing. A clear correlation between intensities of the charge states $\mathrm{Ti}^{1+}$ and $\mathrm{Ti}^{2+}, \mathrm{Ti}^{2+}$ and $\mathrm{Ti}^{3+}, \mathrm{Ti}^{3+}$ and $\mathrm{Ti}^{4+}$ can be seen in Figure 4, which indicates that the formation of $\mathrm{Ti}^{n+}$ most probably occur through ionization of $\mathrm{Ti}^{(n-1)+}$ by the impact of the fast electrons or by multi-photon ionization rather than the direct processes. Similar correlation between various charge states of $\mathrm{W}$ and $\mathrm{Cu}$ ions produced by nanosecond pulsed laser ablation has already been reported (Ilyas et al., 2011; 2012).

In order to estimate energy distribution of each charge state, large number of TOF-EEA spectra were recorded in a wide range of filtering criteria $E / q=72-1650 \mathrm{eV} /$ charge by varying back plate voltage of EEA. Laser fluence was kept fixed at $20.3 \mathrm{~J} / \mathrm{cm}^{2}$ during this experiment. These

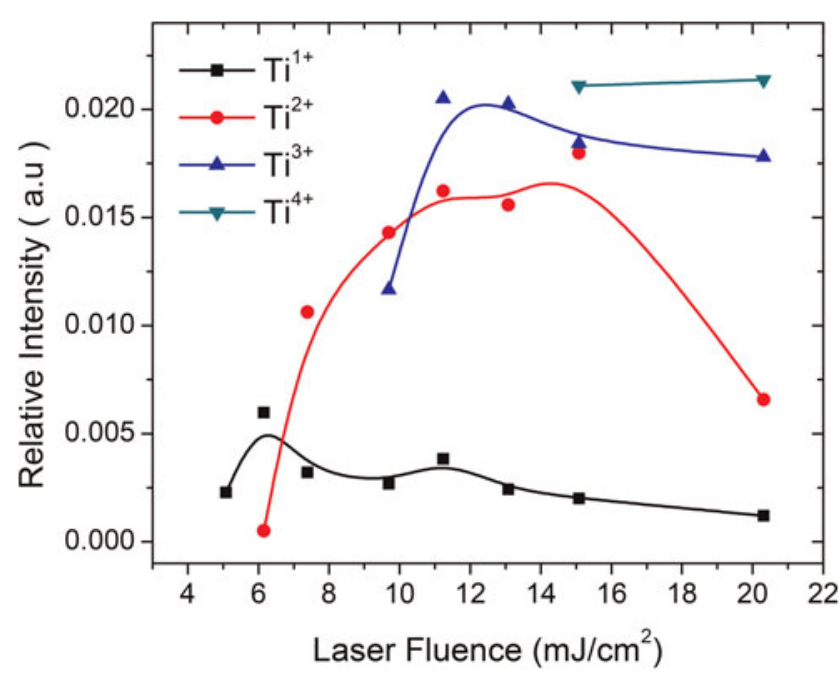

Fig. 4. Intensity of various ion charge states as a function of laser fluence.

spectra permit to obtain a plot between amplitude of ion signal and ion energy for different charge states recognized in TOF-EEA spectra. This method is explained in detail elsewhere (Woryna et al., 1996). Ion intensity as a function of ion energy for four Ti charge states is given in Figure 5. It can be seen that $\mathrm{Ti}$ ions have energies in the range of 0.36-3.0 keV. The energy range of $\mathrm{Ti}^{1+}, \mathrm{Ti}^{2+}, \mathrm{Ti}^{3+}, \mathrm{Ti}^{4+}$ is $0.36-1.72 \mathrm{keV}, \quad 0.68-2.65 \mathrm{keV}, \quad 1.13-2.77 \mathrm{keV}$, $1.45-2.98 \mathrm{keV}$, respectively, which indicates that range of ion energy shifted to higher values with the increase of charge state. Most probable ion energy (peak ion energy) of each charge state obtained by fitting the experimental data of Figure 5 is plotted as a function of charge state in Figure 6. It shows that ion energy is linearly proportional to the charge state, which is clear evidence that an equivalent potential, $V_{o}$, exists inside the non-equilibrium plasma

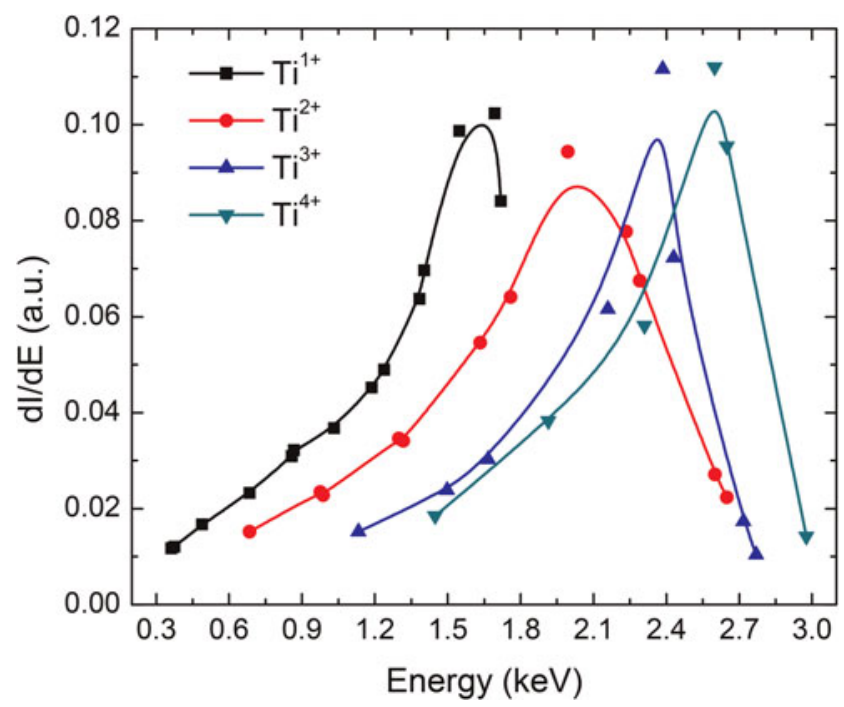

Fig. 5. Energy distribution of various Ti ion charge states measured for a fixed laser fluence of $20.3 \mathrm{~J} / \mathrm{cm}^{2}$. 


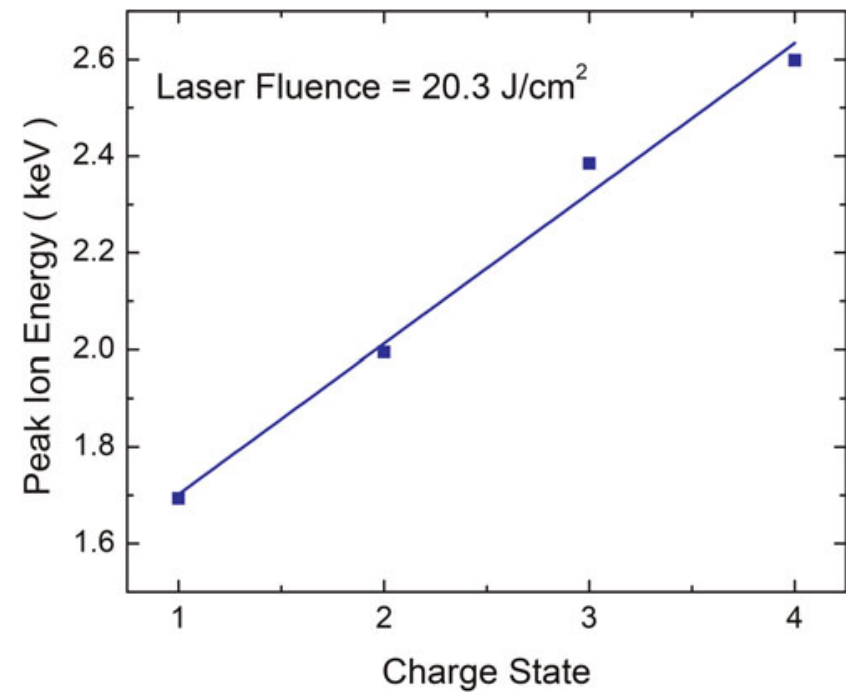

Fig. 6. Peak ion energy as a function of the charge state for fixed laser fluence of $20.3 \mathrm{~J} / \mathrm{cm}^{2}$.

produced by laser ablation. A straight line $y=m x+C$ is fitted to the experimental data shown in Figure 6, where slope $m=e V_{o}$ and parameter $C$ gives non-Columbian contribution of the ion energy. It is already reported nonColombian ion energy is due to thermal and adiabatic processes (Kelly, 1992; Margarone, 2007). The equivalent potential obtained from the slope of the straight line is $310 \mathrm{~V}$. Previously, equivalent potential equal to $270 \mathrm{~V}$ (Torrisi et al., 2008b) and $333 \mathrm{~V}$ (Margarone, 2007) has been reported for the Ti plasma produced by the laser energy of $270 \mathrm{~mJ}$ and Ni plasma produced by laser energy of $300 \mathrm{~mJ}$, respectively. The results reported in this paper are in good agreement with predictions of electrostatic model (Demtröder et al., 1970), which is usually used to explain the ion acceleration in laser plasma. According to this model the electrons heated by the laser photons via inverse bremsstrahlung transfer energy to ions on the time scale of the electron ion thermalization time. As this time is much shorter than the laser pulse duration, electrons and ions obtain equal thermal energies but due to the huge mass difference the electron velocities are much higher than that of the ions. As a result electrons escape the plasma much early and generate an electrostatic field, which accelerates ions proportional to their charge state.

\section{CONCLUSION}

Ti plasma produced by $1064 \mathrm{~nm} \mathrm{Nd:YAG} \mathrm{laser} \mathrm{have} \mathrm{been} \mathrm{in-}$ vestigated using ion collector and TOF electrostatic energy analyzer. Ti ions charge state distributions as a function of laser fluence has been obtained. It has been shown that maximum available charge state increases with the laser fluence and threshold fluence for appearance of a specific charge state can be measured. The observed correlation between relative abundance of various charge states points towards the stepwise ionization of highly charged ions. It is also noted that the laser fluence can be used to control the charge state distribution of the plasma. Indicating that in a practical laser-based ion source, ion charge of interest can be maximized by the variation of laser fluence. The energy distribution of various Ti ion charge states and equivalent potential inside the plasma has been obtained. We believe that these experimental results will provide valuable insight into laser produced metal plasmas.

\section{REFERENCES}

Burdt, R.A., Tao, Y., Tillack, M.S., Yuspeh, S., Shaikh, N.M., FlaXer, E. \& NaJMabadi, F. (2010). Laser wavelength effects on the charge state resolved ion energy distributions from laserproduced Sn plasma. J. Appl. Phys. 107, 043303-043307.

CAridi, F., Torrisi, L. \& Giuffrida, L. (2010). Time-of-flight and UV spectroscopy characterization of laser-generated plasma. Nucl. Instr. Meth. B 268, 499-505.

Cho, N.I., Chol, Y., Lee, S.J. \& LeE, D.Y. (2006). Barrier property of a TiN layer prepared by using flow modulation CVD for $\mathrm{Cu}$ metallization. J. Korean Phys. Soc. 48, 1620-1623.

Collier, J., Hall, G., Haseroth, H., Kugler, H., Kuttenberger, A., Langbein, K., Scrivens, R., Sherwood, T., Tambini, J., Sharkov, B., Shumshurov, A. \& MAsek, K. (1996). The CERN laser-ion source. Laser Part. Beams 14, 283-292.

DEMTRÖDER, W. \& JANTZ, W. (1970). Investigation of laser-produced plasmas from metal-surfaces. Plasma Phys. 12, 691-703.

Haseroth, H. \& Hora, H. (1996). Physical mechanisms leading to high currents of highly charged ions in laser-driven ion sources. Laser Part. Beams 14, 393-438.

Ilyas, B., Dogar, A.H., Ullah, S. \& QAyyum, A. (2011). Laser fluence effects on ion emission from a laser-generated $\mathrm{Cu}$ plasma. J. Phys. D: Appl. Phys. 44, 295202-295208.

Ilyas, B., Dogar, A.H., Ullah, S., Mahmood, N. \& Qayyum, A. (2012). Multiply charged ion emission from laser produced tungsten plasma. Laser Part. Beams 30, 651-657.

Iтон, Y., Ітон, А., Azuma, H. \& Hiокі, T. (1999). Improving the tribological properties of Ti-6Al-4 V alloy by nitrogen-ion implantation. Surf. Coat. Technol. 111, 172-176.

Jordan, R., Cole, D., Lunney, J.G., Mackay, K. \& Givord, D. (1995). Pulsed laser deposition of particulate-free thin films using a curved magnetic filter. Appl. Surf. Sci. 86, 24-28.

Krasa, J., Laska, L., Maisek, K., Pfeifer, M., Kralikova, B., Skala, J., Straka, P., Rohlena, K., Mroz, W., Woryna, E., Parys, T.P., Wolowski, J., Haseroth, F.H., Golubev, A.A. \& Sharkov, B.YU. (1998). Multiply charged ions from iodine laser-produced plasma of medium and high $\mathrm{Z}$ targets. Laser Part. Beams 16, 5-12.

Komotori, J., Lee, B. J., Dong, H. \& Dearnley, P.A. (2001). Corrosion response of surface engineered titanium alloys damaged by prior abrasion. Wear 251, 1239

Kobayashi, M., Funami, K., Suzuki, S. \& Ouchi, C. (1998). Manufacturing process and mechanical properties of fine TiB dispersed Ti-6Al-4 V alloy composites obtained by reaction sintering. Mater. Sci. Eng. A 243, 279-284.

Kelly, R. (1992). Gas dynamics of the pulsed emission of a perfect gas with applications to laser sputtering and to nozzle expansion. Phys. Rev. A 46, 860-874. 
Láska, L., Krása, J., Mašek, K., Pfeifer, M., Trenda, P., Králiková, B., Skála, J., Rolena, K., Woryna, E., Farny, J., Parys, P., WoŁowski, J., Mróz, W., Shumshurov, A., Sharkov, B., Collier, J., Langbein, K. \& Haseroth, H. (1996). Multiply charged ion generation from NIR and visible laser-produced plasma. Rev. Sci. Instum. 67, 950-952.

LiNDL, J. (1995). Development of the indirect-drive approach to inertial confinement fusion and the target physics basis for ignition and gain. Phys. Plasmas 2, 3933-4024.

LunNey, J.G. (1995). Pulsed laser deposition of metal and metal multilayer films. Appl. Surf. Sci. 86, 79-85.

LeE, J.H. \& Hangbo, C.K. (2005). Characterization of the optical and structural properties for low-emissivity filters with Ti, TiOx, and ITO barrier layers. J. Korean Phys. Soc. 46, 154-158.

Mannion, P.T., Favre, S., O’Connor, G.M., Doggett, B., Lunney, J.G. \& GLYNN, T.J. (2005). Langmuir probe study of plasma expansion in femtosecond pulsed laser ablation of silver. Proc. of SPIE 5827, 457-466.

Margarone, D. (2007). Ion acceleration and diagnostics in lasergenerated plasmas. Ph.D thesis, Italy, Università degli Studi di Messina.

Mostako, A.T.T. \& Khare, A. (2012). Molybdenum thin films via pulsed laser deposition technique for first mirror application. Laser Part. Beams 30, 559-567.

Nassisi, V., Pedone, A. \& Rainò, A. (2002). Preliminary experimental results on an excimer laser ion source. Nucl. Instr. Meth. B 188, 261-267.
Riabinina, D., Irissou, E., Drogoff, B.L., Chaker, M. \& Duay, D. (2010). Influence of pressure on the Pt nanoparticle growth during PLD. J. Appl. Phys. 108, 034322-034326.

Sharkov, B. (1995). Handbook of Ion Sources. Boca Raton: Chemical Rubber, p. 149.

Torrisi, L., Gammino, S., Andò, L., Nassisi, V., Doria, D. \& Pedone, A. (2003). Comparison of nanosecond laser ablation at 1064 and $308 \mathrm{~nm}$ wavelength. Appl. Surf. Sci. 210, 262-263.

Torrisi, L., Caridi, F., Margarone, D. \& Giuffrida, L. (2008). Plasma-laser characterization by electrostatic mass quadrupole analyzer. Nucl. Instr. Meth. B 266, 308-315.

Torrisi, L., Margarone, D., Borrielli, A. \& Caridi, F. (2008). Ion and photon emission from laser-generated titanium-plasma. Appl. Surf. Sci. 254, 4007-4012.

Trtica, M., Batani, D., Redaelli, R., Limpouch, J., KmetiK, V., Ciganovic, J., Stasic, J., Gakovic, B. \& Momcilovic, M. (2013). Titanium surface modification using femtosecond laser with $10^{13}$. $10^{15} \mathrm{~W} / \mathrm{cm}^{2}$ intensity in vacuum. Laser Part. Beams 31, 29-36.

Vlădoiu, I., Stafe, M., Neguţu, C. \& Popescu, I.M. (2008). Nanopulsed ablation rate of metals dependence on the laser fluence and wavelength in atmospheric air. U.P.B. Sci. Bull., Series A 70, 119-126.

Woryna, E., Parys, P., Wolowski, J. \& Mroz, W. (1996). Corpuscular diagnostics and processing methods applied in investigations of laser-produced plasma as a source of highly ionized ions. Laser Part. Beams 14, 293-331.

Yeates, P., Costello, J.T. \& Kennedy, E.T. (2010). The DCU laser ion source. Rev. Sci. Instum. 81, 043301-043310. 